\title{
ELEMENTOS PARA EVALUAR PLANES DE ESTUDIO EN LA EDUCACIÓN SUPERIOR
}

\author{
Leda María Roldán Santamaría \\ Catedrática de la Universidad de Costa Rica.
}

Recibido 14-VII-2004

- Aceptado 10-VIII-2004

\section{Introducción}

El establecimiento de un plan de estudios para el desarrollo de una carrera a nivel universitario o la actualización de uno ya vigente, obliga a pensar en su evaluación, lo cual favorece la confiabilidad y validez del plan y de la carrera que respalda. El evaluar un plan de estudios permite descubrir qué aspecto es necesario actualizar, los aciertos, las fallas, las debilidades y las actualizaciones necesarias que se requieren para ponerlo acorde con el desarrollo científico y tecnológico y con las demandas de la sociedad a la que servirá el profesional que se forme con ese plan de estudios.

Esa necesidad de revisión y de actualización de los planes de estudio para carreras de educación superior es motivo para proponer un modelo que sirva para evaluar los planes de estudio de la Universidad de Costa Rica, ante la necesidad de que la práctica curricular responda a una realidad nacional, a una actualización de acuerdo con el avance que experimenta la sociedad a la cual debe su existencia y que sea coherente con las necesidades de formación que esa sociedad demanda.

En este artículo, que resulta ser la recopilación de criterios y evaluaciones de planes aplicados en otras latitudes y ajustado a la institución que interesa, se presentan los lineamientos para generar una propuesta de evaluación de un plan 


\begin{abstract}
This article presents different elements and guidelines to generate a curriculum evaluation of a study plan in the higher education. It identifies the importance that this process has for the institution with the purpose of analyzing the weaknesses and strengths to make decisions for its updating. One finds the answers to why and what for evaluating is necessary and the benefits the users will receive with such updating, the definition of the elements of the study plan that is going to be evaluated and the definition of the variables that will be applied to the evaluation of internal and external elements. Besides, the social sectors involved in evaluation are proposed, the correct moment to evaluate is suggested as well as how often the evaluation must take place. Finally, an evaluation plan is established so that the plan does not become outdated.
\end{abstract}

Key words: Validation, curriculum, Higher Education. de estudios en la educación superior. Se toma en cuenta la importancia que tiene, para la institución educativa, conocer las debilidades y fortalezas del plan con miras a la toma de decisiones para ponerlo al día. En la propuesta se responde el por qué y el para qué se debe evaluar ese plan de estudios; así mismo se habla de los beneficios que los usuarios recibirán de esa actualización; de la concreción de elementos del plan de estudios por evaluar; de la definición de las variables que se aplicarán en la evaluación de los elementos externos e internos; a su vez se proponen los sectores sociales involucrados en la evaluación; y se analiza el momento adecuado para realizar la evaluación y cada cuánto tiempo debe hacerse; también se establece un plan estratégico para realizar la evaluación y se presentan las sugerencias de los usos que se le pueda dar a la información recopilada con ella.

La primera parte del artículo presenta la fundamentación teórica sobre el que se basa la presente propuesta, y en la segunda parte se establecen los lineamientos y sugerencias para poner en práctica un modelo de evaluación de un plan de estudios.

\subsection{Justificación e importancia}

La evaluación de un plan de estudios es necesario realizarla porque, al avanzar el desarrollo científico y tecnológico, los planes de estudio se desactualizan. Pueden no responder a las necesidades que genera el cambio socio-cultural y quedan fuera de la realidad de acuerdo con las necesidades que la sociedad requiere satisfacer.

$\mathrm{Al}$ estar inmersos dentro de un mundo en constante cambio, los factores de dinamismo económico y los procesos sociales obligan a realizar ajustes en los planes y en los programas de formación de profesionales. Estos ajustes permiten enfrentar y proponer los cambios que requiere la sociedad favorecida. Por eso, es necesario contar con planes de estudio que se caractericen por ser dinámicos y que respondan a las necesidades sociales e individuales. De este 
dinamismo, también debe quedar evidencia al proponer la práctica de nuevas teorías de aprendizaje, así como la utilización de tecnologías modernas y de metodologías acordes con los avances en el campo educativo, que favorezcan los planes de estudio que forman parte del currículum institucional de la Universidad de Costa Rica.

La importancia de evaluar un plan de estudios radica en que, permite descubrir qué cambios son necesarios para un rediseño de los planes, el establecimiento de los lineamientos para su actualización y el tiempo en que se debe cumplir con esta para que el plan no pierda vigencia.

Cuando se piensa en la evaluación del plan de estudios se debe involucrar a la población beneficiada; a los formadores y a la sociedad en general, la cual puede estar representada en los profesionales graduados de ese plan de estudios, en los empleadores y en los beneficiados primarios de la labor de esos profesionales. Son todos ellos los que se favorecen con los profesionales que se formen con ese plan de estudios. Es la sociedad, aquellas personas que se benefician directamente de esos profesionales, la que debe identificar cuáles son sus necesidades y cómo espera que se satisfagan.

Un plan de estudios vigente se propone para satisfacer las necesidades de una sociedad que requiere un profesional en un campo específico; de ahí que, se requiere verificar que ese plan se esté ejecutando de acuerdo con las demandas de un grupo específico de la sociedad, que los contenidos de los cursos sean apropiados al perfil profesional, que el nivel de exigencia corresponda con el tipo de profesional, que la formación integral que se está impartiendo sea la que la sociedad requiere, que la formación en valores y en ejes transversales cumpla con las exigencias morales y éticas de la sociedad a la que ese profesional va a servir.

Considerando el acelerado avance en los distintos campos del conocimiento científico-tecnológico, es necesario que los planes de estudio se actualicen con regularidad y que conduzcan, además, a una formación continua fuera de las aulas universitarias.

\subsection{Referencial orientador para la evaluación}

La Universidad de Costa Rica como formadora de profesionales tiene la misión de revisar y reevaluar su quehacer académico, con el fin de propiciar el avance en el conocimiento para lograr la máxima expresión y llenar así las necesidades de la sociedad costarricense.

La rapidez con que avanza el conocimiento, y la misión que debe cumplir la Universidad exige que esta adecue su estructura de organización académica, y así hacer frente al reto que significa formar profesionales para el bien común con excelencia académica y con una capacidad y un conocimiento idóneo para afrontar los retos de un mundo altamente cambiante y competitivo.

En la modernización de su estructura, la Universidad cuenta con Vicerrectorías encargadas de velar por los diferentes campos del quehacer universitario. Le corresponde a la Vicerrectoría de Docencia, por su parte, velar por la actualización de los planes de estudio. Esto lo contempla el Estatuto Orgánico en el capítulo 5, artículo 50, en los que se indica lo siguiente:

“a) Sancionar y supervisar los diversos planes de estudio de la Universidad, con el propósito de coordinarlos en lo posible y adaptarlos a las necesidades de interés nacional.

b) Velar porque la labor docente en la Universidad se lleve a cabo en forma eficiente y actualizada, en unidad de propósitos con la investigación, utilizando los sistemas más adecuados de enseñanza y evaluación.” (UCR, 1998, p. 14).

Como parte de sus funciones esta Vicerrectoría debe velar por la administración y supervisión de los planes de estudio y sus correspondientes programas. Por otra parte, además, le ha correspondido emitir resoluciones relacionadas con 
el planteamiento, la evaluación, el control y las modificaciones de los mismos, de acuerdo con los lineamientos de la reglamentación universitaria establecida en su Estatuto Orgánico y las directrices que dicta CONARE.

En el documento sobre "Políticas y Normas Curriculares para la Actualización de Planes de Estudio" (1995) emitido por la Vicerrectoría de Docencia, y en el currículum de la Universidad de Costa Rica es posible establecer tres niveles esenciales. El primero de ellos es el orientador, que comprende los principios, propósitos y políticas institucionales; el segundo corresponde a las regulaciones establecidas en la normativa, donde se incluyen los reglamentos y resoluciones, y el tercero, comprende los planes y programas de estudio en los que se definen los aprendizajes que los futuros profesionales deben lograr para conseguir un grado académico.

Con base en el tercer nivel es que se debe proponer la evaluación de los planes de estudio para la educación superior, lo que permite comprobar si cumplen con las expectativas de formación del profesional que la sociedad costarricense requiere y que la Universidad debe formar.

La evaluación de un plan de estudios debe identificarse como una investigación en el cual se requiere la participación de toda la comunidad educativa, y debe conducir al conocimiento de sus fortalezas y debilidades; esto, con el fin de establecer juicios de valor que conduzcan a propuestas que mejoren los planes, con el propósito de satisfacer las necesidades sociales y de formación académica.

Referida a cualquier ámbito, la evaluación puede ser conceptualizada como un proceso mediante el cual se recoge, analiza e interpreta información relativa a una determinada actividad, con el objeto de emitir juicios y facilitar la toma de decisiones en términos de ajustes temáticos, reorientación de objetivos, reformulación de perfiles, selección y reorganización de recursos, entre otros temas. Así, la evaluación de un plan de estudios consiste en reconocer sus fallos y aciertos, ventajas y desventajas. Principalmente, la tarea es plantear alternativas viables de reorientación de las acciones y de los trabajos de quienes deben aplicar dicho plan de estudios. Es una oportunidad de unificar criterios que permitan establecer los lineamientos para la transformación cualitativa de los planes de estudio. Esto es, que se fundamenten en los cambios que favorezcan a la sociedad, fortaleciendo su formación cultural. Se presenta la posibilidad de innovación al considerar las nuevas metodologías y tecnologías facilitadoras del proceso de aprendizaje y el surgimiento de proyectos que permitan desarrollar los planes de estudio para que los estudiantes se vean favorecidos en su evolución.

Esa evaluación debe ser un proceso participativo que obtenga y analice información útil con el propósito de juzgar y tomar decisiones alternativas, respecto de la concepción, estructura, funcionamiento y administración de los planes de estudios. En este sentido, se puede afirmar que es un proceso participativo, porque debe tomar en cuenta a todos los actores que intervienen en la ejecución de ese plan, como son los estudiantes, profesores, directores, representantes de la sociedad civil, egresados, gremios, empleadores y administradores. Así, la evaluación se concibe dentro de parámetros cuantitativos y cualitativos para poder juzgar el grado de logro; así como también sus eventuales deficiencias y de este modo, tomar las decisiones relativas a los ajustes, a la reformulación o a los cambios que deben ejecutarse; igualmente, permite verificar la productividad, la eficacia y la pertinencia del plan de estudios en cuestión.

La evaluación se emprende como una acción intencionada que debe obedecer a un proyecto de investigación evaluativa, centrada en los campos o ejes que interesa evaluar y que son definidos según las necesidades de los actores sociales: administrativos, docentes, estudiantes, empleadores, entre otros. La determinación de 
los diferentes procesos y factores que se pretenden evaluar son una etapa muy importante y necesaria.

La actividad evaluativa debe ser comprendida, además, como sistemática y permanente, de manera que permita mejorar en forma continua ese plan. Este puede dejar de responder a las necesidades y valores que lo justifican, no cumpliendo con la satisfacción de las necesidades del contexto social y cultural, que demanda el avance del conocimiento científico y tecnológico y de las necesidades sociales que se generan al paso del tiempo.

\subsubsection{Confiabilidad y validez de la evaluación}

Es importante resaltar que este proceso debe fundamentarse en criterios de adecuación científica, tales como la validez y la confiabilidad, tanto de las técnicas como de los instrumentos que se empleen. Así mismo, deben de estar presentes en cada una de las etapas del desarrollo curricular como lo es la planificación, la supervisión, la instrumentación, la ejecución y la propia evaluación, todo con el fin de propiciar la calidad de cada una de ellas.

La Universidad de Costa Rica, en general, y la Vicerrectoría de Docencia, en particular, deben considerar que la evaluación de un plan de estudios obedece a las siguientes premisas propuestas por Luis Sime (1999, p. 4):

“a- $\quad$ No es un fin en sí misma, sino que adquiere su sentido en la medida en que apoye el desarrollo de este nivel educativo.

b- $\quad$ Debe ser parte integral de los procesos de planeación de las tareas académicas y de apoyo, y no un proceso superpuesto para dar cumplimiento a requerimientos administrativos.

c- Debe entenderse como un proceso permanente que permita mejorar de manera gradual la calidad académica, entendida esta como aquel proceso que permite cumplir con el plan de estudios a satisfacción de los usuarios. En consecuencia debe incorporar una visión a lo largo del tiempo, que permita evaluar avances y logros, identificar obstáculos y promover acciones de mejoramiento académico.

d- Los procesos de evaluación de los planes de estudio actuales y aquellos que se generen, deben incidir en la formación profesional y en el desarrollo de la educación en campos como la Ciencia, la Tecnología y aquellos que contribuyen al desarrollo social y cultural del país."

Ante esta posición, es necesario generar nuevos conocimientos referidos al desarrollo de los planes y sus programas que indiquen las eventuales modificaciones y adaptaciones necesarias que deben realizarse para que los planes de estudio atiendan las necesidades individuales del estudiante y del sistema social.

Según la Vicerrectoría de Docencia y el Estatuto Orgánico de la Universidad de Costa Rica:

"la práctica curricular de la Universidad de Costa Rica se caracteriza por el predominio del enfoque académico y por la diversidad en los diseños de los planes de estudio. En estos se manifiestan diversos niveles de articulación entre teoría y realidad; sin embargo, desde fines de la década del setenta se observa una tendencia creciente a dar énfasis al hacer sobre el comprender y el explicar, producto de la influencia de los enfoques tecnológicos." (Vicerrectoría de Docencia, 1995, p. 9).

Es en el nivel operativo, donde la Vicerrectoría tiene un papel protagónico en relación con la evaluación y el currículum universitario. La Vicerrectoría de Docencia tiene dentro de sus objetivos atender las modificaciones que deben aplicarse a los planes de estudio. Es a partir de esta problemática que dicha Vicerrectoría debe proponer la evaluación de los diferentes planes y su actualización, tratando de que se ajusten más a la política curricular que establece el Estatuto Orgánico y aquellas que corresponden a las unidades académicas. Dentro de las normas curriculares que la Vicerrectoría establece para tener los planes de estudio actualizados se puede mencionar lo siguiente:

- $\quad$ "Deben responder al compromiso de la Universidad con la sociedad, con planes 
de estudio que atiendan al conocimiento científico y tecnológico actualizado, que se vinculen con el mejoramiento de la calidad de vida de la población y que contemplen las necesidades del desarrollo sostenible, considerando la demanda de los sectores productivos, sociales y profesionales.

- Deben propiciar la relación entre teoría y práctica, aplicando metodologías que favorezcan una reflexión crítica del ejercicio profesional, que a su vez permitan ejercer acciones docentes acompañadas de metodologías de investigación desde los primeros niveles de formación, y que incluyan programas y proyectos de acción social vinculados con sectores relacionados con el campo profesional que se estudia, y que además faciliten la inclusión de temáticas articuladoras de la sostenibilidad con la identidad cultural.

- Deben ser planes de estudio que presenten estructuras de organización flexibles, que incorporen una visión humanista a partir de los problemas específicos que se afrontan en cada área del conocimiento, y que estos se distribuyan a lo largo de todo el plan, ofreciendo diferentes alternativas de cursos de servicio y optativos equivalentes para una misma área.

- Deben ser más coherentes, fundamentados en los principios y propósitos del Estatuto Orgánico, y que integren actividades de docencia, investigación y acción social de acuerdo con el enfoque curricular asumido.

- Deben propiciar una adecuada retroalimentación, de forma que la evaluación sistemática sea coherente con los principios en que se fundamenta el plan, que contemple diferentes modalidades de evaluación y que se propicien acciones conducentes a la autoevaluación en la que participe toda la comunidad." (Vicerrectoría de Docencia, 1995, p. 6).

\subsubsection{Concepto de evaluación}

Autores como Díaz Barriga, (1991, p. 45), presentan sus conceptos de evaluación, según el objetivo que se persiga al aplicarla y presentan diferencias, según el énfasis que se establezca en sus diferentes elementos. El concepto que mejor se ajusta a la evaluación que nos ocupa es el de Alicia de Alba, el cual se refiere a los elementos que deben incluirse en la evaluación de los planes y programas. De esta manera:

“... es posible concebir al campo de la evaluación, como investigación evaluativa, en la medida en que en toda tarea de evaluación se conjugan aspectos teóricos (vinculados con la producción de conocimiento) y aspectos axiológicos (vinculados con la valoración y el compromiso), debido a los aspectos teóricos que desde nuestra posición, son inherentes a todo proceso de evaluación." (De Alba; 1991, p. 93).

De esta manera, la evaluación debe ser un proceso integral que contemple la teoría, la práctica y las valoraciones; en lo cual se fundamenta la investigación para emitir juicios de valor acerca de la pertinencia de todos y cada uno de los factores del diseño, rediseño e implementación del plan de estudios; elemento que conduce a la construcción de conocimientos con el fin de mejorar el o los elementos que puedan ser evaluados.

Según Alicia de Alba la evaluación debe ser integral, de modo que contemple la teoría, la práctica y los aspectos axiológicos. "Se ha definido la evaluación como un proceso en el cual juegan un papel central la comprensión (teórica) y la valoración axiológica. (De Alba; 1991:105)”. Para esta propuesta de evaluación de un plan de estudios vigente en la Universidad de Costa Rica, la evaluación debe involucrar los aspectos que contemplan las normativas universitarias pertinentes, lo que realmente se le ofrece a los estudiantes de dicho plan y la formación 
que estos estudiantes logran al finalizar el plan de la carrera que optaron.

También, Glazman y De Ibarrolle, citados por Peraza, presentan la evaluación del plan de estudios como:

“... un proceso objetivo y continuo, que se desarrolle en espiral, y consiste en comparar la realidad (objetivos y estructura del plan vigente), con un modelo, de manera que los juicios de valor que se obtengan de esta comparación, actúen como información retroalimentadora que permite el adecuar el plan de estudios a la realidad, o cambiar aspectos de esta." (Peraza, 1998, p. 3).

\subsubsection{Definición de plan de estudios}

Por plan de estudios se entiende una estructura que orienta a la comunidad educativa y manifiesta la planificación del proceso de aprendizaje. De acuerdo con Carvajal (1984:64) "Es una síntesis instrumental mediante la cual se organizan y ordenan una serie de factores tales como propósitos, metas, disciplinas, recursos y perfiles, para fines de enseñanza y aprendizaje de una profesión que se considere social y culturalmente importante."

El plan de estudios persigue como objetivo, presentar un orden lógico de factores que afectan la formación profesional e integral de una persona, que debe reunir ciertas características acordes con las necesidades sociales. En ese ordenamiento lógico se incluyen los diferentes cursos con los que debe cumplir un estudiante para lograr su formación profesional; cursos que deben tener contenidos seleccionados para dar una formación integral al individuo, y que este construya su conocimiento sobre un campo epistemológico específico; además, deben considerar las horas que debe dedicar a cada curso, el orden en que debe cumplir cada uno, para que reúna los requisitos y correquisitos de los siguientes. La aprobación de esos cursos conduce al estudiante a la obtención de un título universitario que lo acredita a ejercer la profesión que eligió. Así mismo, se debe incluir el perfil profesional y las expectativas de desenvolvimiento laboral que se espera de ese profesional.

Ese plan de estudios se puede ubicar en una malla curricular, la cual se entiende como "aquella estructura secuencial de asignaturas lectivas y prácticas que forman un programa de estudios, en la que se señalan sus requisitos, duración y objetivos". (UNESCO, 2001:1). Otros autores, como Díaz, manifiestan que el mapa curricular "está constituido por la descripción sintética de contenidos de cada una de las asignaturas que forman el plan de estudios." (Díaz; 1997, p. 42).

El plan de estudios de un sistema educativo está íntimamente relacionado con los propósitos de ese sistema, el tipo de necesidades sociales o individuales que se consideraron en su elaboración, las áreas de formación en que está organizado y las nociones básicas de cada una de dichas áreas; eso con el fin de obtener un plan de estudios que permita visualizar la forma en que se apoyan e integran los diferentes contenidos de las asignaturas, garantizando de ese modo, que no se repitan contenidos y que se integren los aprendizajes.

Le corresponde a la Vicerrectoría de Docencia de la Universidad de Costa Rica tener presente estos planes de estudio, ya que se le designa:

"Velar porque los cursos universitarios presten especial cuidado a la vinculación de los conocimientos universales con la realidad nacional, desarrollando en el estudiante una capacidad de análisis y de crítica que le permita aplicar creativamente los conocimientos adquiridos.” (Del Vechhio; 1986, p. 6).

El Centro de Evaluación Académica (CEA) de la Universidad de Costa Rica, establece lo que se concibe como plan de estudios de una carrera y los diferentes elementos que la conforman:

"La estructura de cursos del plan de estudios debe ser respuesta a los planteamientos obtenidos mediante el perfil profesional y con base en la realidad y necesidades futuras del país. Constituye en orden armónico, el listado en que se consignan las características de cada curso, a saber, nivel, sigla, nombre, período, 
cursos requisitos, cursos correquisitos, horas por semana y créditos.” (CEA; 1991, p. 47).

El plan de estudios debe estar sometido a procesos de evaluación que contemplen todos los elementos que lo conforman.

\subsection{Factores que debe contemplar el modelo de evaluación}

La propuesta de un modelo de evaluación para un plan de estudios en la educación superior, obliga a definir cuales son los objetos del plan de estudios que se desean evaluar.

Como primer paso de la evaluación, debe establecerse el modelo ideal de lo que debe ser ese plan de estudios, con base en el perfil del profesional que se desea graduar. Ese modelo debe tener una fundamentación teórica que lo respalde, la cual surge de las teorías modernas sobre el aprendizaje y la metodología de la especialidad. Lo cual conduce a una comparación teórica contra la realidad de lo que se tiene.

Cuando el modelo del plan se tiene escrito, es necesario analizar los objetivos, si estos contemplan las necesidades del plan de estudios vigente, y si cumplen con las expectativas de la sociedad, a la cual sirve el profesional que se formó con dicho plan.

Los objetivos se logran cumplir en la medida en que, la estructura del plan se consolide para satisfacer las necesidades de los usuarios; que estos logren aprobar los cursos en el tiempo previsto, que se les satisfagan las necesidades de matrícula y que el nivel de exigencia este acorde con el nivel de evaluación de los cursos que lo integran.

Los objetivos de un plan de estudios conducen a un perfil profesional propuesto a partir de las necesidades socio-culturales. Es necesario verificar si ese perfil está actualizado, si cumple con las necesidades por satisfacer en una comunidad o si requiere de un planteamiento nuevo, en razón de los avances sociales, económicos, científicos y tecnológicos.
Por lo antes expuesto, es necesario establecer áreas o tipos de evaluación dentro del modelo propuesto para evaluar un plan de estudios. Una forma simple de lograr el establecimiento de dichas áreas, consiste en agrupar los objetos de evaluación en dos tipos de relaciones significativas: la congruencia interna y la congruencia externa.

En la congruencia interna se contempla la relación entre los distintos elementos del plan de estudios, y en la congruencia externa se considera la relación del plan de estudios con el contexto social.

\subsubsection{Elementos pertinentes con la congruencia interna de un plan de estudios}

La evaluación de la congruencia interna de los planes de estudio, contempla aspectos como:

- Los objetivos generales del plan, confrontados con los fundamentos. Esto permite descubrir si el plan tiene faltante de cursos u omisiones de otros, si se dan repeticiones o si la organización del plan debe modificarse.

- La viabilidad del plan en relación con los recursos, tanto materiales como humanos, y si son suficientes para cumplir los objetivos propuestos. Es necesario verificar si los recursos son accesibles a los profesores y a los estudiantes. Aquí, se entiende por recursos humanos, los profesores y el personal administrativo; y por recursos materiales, se consideran las aulas, libros, laboratorios, medios audiovisuales y material didáctico en general.

- La continuidad e integración del plan, la cual confronta los objetivos de los cursos con los objetivos del plan. Es necesario, verificar si los cursos 
ofrecen las necesidades de formación, y si por medio de los objetivos de los cursos es posible lograr los objetivos de la carrera. Por medio de la continuidad, se analiza si la ubicación de los cursos es la apropiada, si la relación entre cursos requisitos y correquisitos presenta la secuencia adecuada.

- $\quad$ El análisis de la interrelación entre los cursos del plan es lo que permite identificar las relaciones congruentes, al comprobar la relación de apoyo entre cursos, y las incongruentes al estar ausente esta relación.

- La vigencia del plan permite identificar su actualidad respecto del perfil profesional, y de los avances en el campo científico, social, disciplinario, psicológico y pedagógico.

Los elementos por considerar en el diseño de los instrumentos que se utilizarán para la evaluación de la congruencia interna de los planes de estudio, pueden ser los siguientes:

- Justificación del plan de estudios, para responder al: ¿por qué de su existencia?, ¿a quién o quiénes favorece?, ¿cuál es su demanda real?, ¿cuál es su pertinencia social?, ¿cuál es su importancia académica?, ¿cómo se enfoca la factibilidad y la viabilidad?

- Recursos disponibles, para responder a las preguntas: ¿son los recursos humanos y materiales disponibles suficientes para los educadores que lo atienden?, ¿cuenta con los recursos académicos necesarios para cumplir con él?

- Infraestructura docente-administrativa, que atiende interrogantes como: ¿existe una comisión compartida que coordina la carrera?, ¿es el coordinador o coordinadora de la carrera una persona accesible a los estudiantes?, ¿se lleva un verdadero expediente donde se controla el avance de los estudiantes?, ¿la oferta de cursos satisface la demanda de los mismos?, ¿satisfacen los horarios de dichos cursos las necesidades de los estudiantes?, ¿la modalidad de los cursos es la adecuada al perfil profesional de la carrera?

- Malla Curricular (estructura del plan), donde se puede responder a: ¿es la estructura del plan la adecuada para cubrir el plan de estudios en el tiempo requerido?, ¿responde la malla curricular con la orientación que tiene el plan de estudios?, ¿brinda ese plan de estudios la formación adecuada a la carrera que pertenece?, ¿los objetivos se cumplen en esa malla curricular?, ¿es la cobertura la adecuada para el ámbito que cubre?, ¿presenta una relevancia y una secuencia adecuadas?

- Modalidades de enseñanza, cuyas preguntas serían: ¿están las metodologías acordes con las demandas de adecuación de los estudiantes de dicho plan?, ¿presenta este plan una flexibilización acorde con las necesidades de los estudiantes y de la sociedad?, ¿la evaluación aplicada responde a las necesidades del plan de estudios?, ¿está el personal docente actualizado en relación con las metodologías y tecnologías modernas que favorecen el proceso de enseñanza y aprendizaje?

\subsubsection{Elementos pertinentes con la congruencia externa de un plan de estudios}

La evaluación de la congruencia externa debe contemplar, principalmente, el impacto social que tiene el egresado de 
ese plan de estudios. Este impacto se puede analizar a partir de elementos como:

- El análisis de las funciones que debe cumplir la persona que se egresa de este plan de estudios, si realmente esta capacitada para hacer frente a las funciones que le demanda el puesto que desempeña, o si es necesario incluir en ese plan la capacitación para otras funciones.

- La revisión de los mercados de trabajo donde se identifican las demandas, las necesidades del campo laboral y los índices de desempleo en este campo. La presencia de mercados laborales novedosos, por ende, obliga a la actualización del plan para satisfacer esos mercados.

- $\quad$ El papel del egresado en la solución de los problemas reales de la comunidad a la cual dedica sus capacidades. Aquí, se identifica si existe o no el vínculo entre la institución educativa y el entorno social.

Los elementos por considerar en el diseño de los instrumentos que se utilizarán para la evaluación de la congruencia externa de los planes de estudio, pueden ser los siguientes:

- Impacto del plan de estudios, que debe dar respuesta a los cuestionamientos: ¿es el número de empadronados equivalente al número de estudiantes activos?, ¿se mantiene el número de estudiantes activos en relación con el número de graduados?, ¿es el tiempo programado para cumplir con este plan de estudios equivalente al tiempo real que le toma a los estudiantes graduarse?, ¿cuántos estudiantes desertan de la carrera?, y ¿qué razones motivan esa deserción?

- Impacto social e institucional, que responde a aspectos como: ¿qué calidad de egresado surge de este plan de estudios?, ¿cómo contribuye este egresado al sistema educativo?, ¿en qué aspectos se favorece la sociedad con este egresado?

- $\quad$ Perfil del egresado, que se plantea: ¿está actualizado el perfil académico respecto del perfil profesional?, ¿responden ambos perfiles a la demanda social y personal?

- Modalidad de actualización permanente, que responde a cuestionamientos como: ¿se incluye la investigación como parte de la metodología y de la evaluación de los cursos del plan de estudios?, ¿se incluyen los nuevos conceptos que esta carrera cubre en la práctica profesional?, ¿cuál es el grado de incorporación de las nuevas tecnologías?, ¿se incluyen dentro de las metodologías de los cursos los procesos de investigación y la participación en eventos relacionados?

La evaluación, tanto de la congruencia interna como de la externa, permite identificar los logros obtenidos a lo largo del desarrollo del plan de estudios. Esto conlleva a la toma de decisiones en cuanto al hecho de continuar presentando el mismo plan de estudios o de modificarlo, para llenar las expectativas de usuarios y de empleadores.

Estas y otras preguntas que surjan en relación con los aspectos en mención, pueden orientar los procesos evolutivos con la finalidad de su actualización o rediseño. No se descarta la aparición de nuevos elementos al analizar las áreas de evaluación que se proponen en el modelo sugerido.

\subsection{Los actores sociales de la evaluación}

Para llevar a cabo la evaluación del plan de estudios, de acuerdo con el modelo propuesto, se deben considerar diferentes 
actores, según el campo que involucre. En cada una de las áreas deben participar todos los actores que forman parte de la acción educativa, como son:

- Director o directora de la carrera, persona que conoce el plan, los usuarios, los departamentos de personal para cubrir en lo que respecta a lo docente, así como los órganos que dictaminen las debilidades y expongan los razonamientos respecto de las necesidades reales.

- Consejo asesor de la unidad académica que administra el plan, quien debe estar al tanto de la demanda que presenta cada curso, el perfil profesional del graduado de dicha carrera y de la demanda que tiene el plan en cuestión.

- Los docentes que imparten los cursos porque son quienes mejor conocen el tipo de estudiante que sigue la carrera, y las necesidades de los cursos que corresponden a ese plan de estudios.

- Los estudiantes que siguen la carrera que corresponde al plan, quienes están al tanto de las necesidades y satisfacciones que brinda, y saben qué aspecto es necesario contemplar en el momento de una actualización.

- El personal administrativo, que tiene conocimiento del plan y colabora con su eficaz aplicación; y porque además conocen las necesidades reales de demanda de los diferentes cursos, tanto de los específicos como de los de servicio.

- La comunidad a la cual beneficia dicho plan, que involucra el contexto social, porque conocen las necesidades comunitarias que ese plan debe satisfacer.

\subsection{Momentos de la evaluación}

Un plan de estudios debe evaluarse en tres etapas, establecidas de acuerdo con la ejecución del plan, contemplando los intereses de la institución que lo tiene en vigencia:

a- Cuando es un plan nuevo, debe evaluarse al finalizar cada ciclo lectivo, para determinar la pertinencia del programa en su orden de cursos por ciclos.

b- Durante la implementación del plan, para analizar la coherencia y la secuencia de los cursos en su orden.

c- Cuando se egresa la primera promoción de la carrera que sigue el plan que se desea evaluar, para establecer si ese plan llenó las expectativas de los profesionales que se graduaron de acuerdo con los contenidos.

Si es un plan que ya está vigente, y que se ha logrado probar que llena las expectativas sociales y personales, debe ser evaluado periódicamente, según las problemáticas identificadas, que tienen sustento en el avance científico, tecnológico, social y económico de la sociedad involucrada. Esto con el fin de poder decidir cuáles son las actualizaciones que el plan requiere.

Según el momento en que se encuentre el plan de estudios, así será el tipo de evaluación que se deba aplicar. La metodología y los aspectos por evaluar se definen de acuerdo con el momento en que se encuentra el plan: si es un plan nuevo, si es un plan de mediano plazo o si es un plan que tiene una larga trayectoria de aplicación.

\subsection{Metodología de la evaluación}

Para la evaluación del plan de estudios se pueden aplicar metodologías participativas en las que el grupo de actores considerados para cada área aporte la 
información necesaria por medio de diferentes técnicas. Los cuestionarios con preguntas cerradas, por ejemplo, permiten una cuantificación más rápida de la información, y dan la oportunidad de generar tablas de datos, que luego se presentan en gráficos que facilitan su interpretación; pero se hace necesario considerar las entrevistas y las observaciones que aportan información más interactiva y de carácter cualitativo.

Las entrevistas abiertas y las entrevistas a profundidad se pueden aplicar a las autoridades, ya que constituyen un número reducido de personas, y pueden aportar información muy valiosa sobre el plan que se evalúa, y confrontar esta con la que aporten otras personas involucradas en el plan de estudios. Dado que es una metodología participativa, es necesario considerar a los estudiantes que reúnen características particulares, como: un excelente rendimiento académico, tiempo récord para graduarse, proyectos de graduación de impacto, entre otros aspectos; que pueden aportar información valiosa y permitir una triangulación con la información que brinden los estudiantes regulares y los estudiantes rezagados del plan.

\subsection{Resultados de la evaluación: su presentación}

Los resultados de los instrumentos aplicados en la evaluación se pueden obtener por medio de tabulaciones extraídas de la información. Para esto, se deben identificar las categorías que se consideraron en la evaluación y los indicadores que permiten hacer un análisis de esa información.

Al identificar las categorías, es posible agrupar la información, según esas categorías, e identificar más fácilmente la que brindan los diferentes instrumentos, de acuerdo con los intereses de la evaluación.

De cada indicador, se obtiene información que permite definir aciertos o fallas del programa en evaluación, asociar los objetivos planteados en la evaluación e identificar si la problemática presentada es real o no.

Una vez obtenidos los resultados por medio de tablas y de gráficos, se procede al análisis de los datos, para identificar los aspectos que se deben sugerir para mejorar el plan de estudios, cuáles deben ser eliminados y cuáles deben ser readecuados a las necesidades de los usuarios.

Los resultados permitirán hacer una presentación justificada de las conclusiones y proponer modificaciones de actualización al plan de estudios.

\section{Síntesis}

Los planteamientos aquí expuestos permiten conocer los aspectos generales que se deben considerar para una evaluación. Son los interesados los que deben estructurar los cuestionarios, las entrevistas a profundidad y escoger la población a la que se le aplicarán. Pero aquí tienen una gruía clara de los aspectos a contemplar para estructurar esos instrumentos.

Los interesados en los procesos evaluativos deben identificar la problemática en un nivel específico y luego diseñar el proceso. Al presentar los elementos para la evaluación de un plan de estudios de educación superior, el objeto es evidenciar todos aquellos aspectos que deben ser tomados en cuenta en el diseño, en la puesta en práctica y en la evaluación del plan, que conduzca a sus usuarios por el camino de una preparación acorde con las necesidades de la sociedad a la cual va a servir.

En el caso particular de la Universidad de Costa Rica, la evaluación de los planes de estudio debe contemplar no solo a los usuarios, sino también a los reguladores de la educación superior costarricense: Consejo Nacional de Rectores (CONARE) y Consejo de Educación Superior Privada (CONESUP). Es necesario considerar las regulaciones emanadas de dichas entidades a la hora de analizar las modificaciones que requiera un plan de estudios. 
La evaluación de los planes de estudio constituye una actividad cuya importancia requiere poner mayor énfasis en los procesos orientados para tal propósito, lo cual supone, sustentar estos procesos tanto desde el punto de vista teórico como desde el metodológico y técnico-Instrumental.

En la Universidad de Costa Rica, se han hecho esfuerzos para aplicar verdaderas evaluaciones, tanto a planes de estudio como a programas y unidades académicas, los cuales han contribuido de manera significativa a mejorar la calidad de los programas de las diferentes carreras que se siguen en dicha casa de estudios; también es cierto, que aún se requieren mayores esfuerzos para profundizar y sistematizar las prácticas y procedimientos evaluativos, con miras a conformar, a mediano plazo, una plataforma y cultura evaluativas que propicien el mejoramiento constante de los planes de estudios, y el mantenimiento de niveles de excelencia acordes con la importancia que revisten para el desarrollo científico, tecnológico y humanístico del país.

\section{Referencias bibliográficas}

Carvajal, Carlos A. "Algunos aspectos teóricos de los planes de estudio". Revista Educación. UCR. Números 1 y 2. Página: 63-69. Costa Rica. 1984.

CEA. Centro de Evaluación Académica. Guía para la elaboración y presentación de modificaciones a planes de estudio. Universidad de Costa Rica.
Vicerrectoría de Docencia. Costa Rica. 1991.

Centro de Evaluación Académica. Guía para la revisión y estructuración de planes de estudio. Universidad de Costa Rica. Vicerrectoría de Docencia. Costa Rica. 1979.

De Alba, Alicia. Curriculum: crisis, mito y perspectivas. México. 1994.

Del Vecchio, Janina. Bases de una reforma curricular. Universidad de Costa Rica. Vicerrectoría de Docencia. San José. Costa Rica. 1986.

Díaz Barriga, Ángel. Didáctica y Currículo. Editorial Paidos. México. 1997.

Peraza, Ernesto. "Proyecto de Evaluación Curricular a los Planes de Estudio en la escuela de Ciencias Sociales". Revista Mar y Arena. México. Noviembre. 1998.

Sime. Luis. "Innovación en la Universidad: aportes para procesos de reforma curricular". Revista Educación. Vol VIII. N¹6. Perú. 1999.

UNESCO. 2001. Definición de plan de estudio. Recuperado el 12 de setiembre de 2002 de www.ibe.unesco. org/Internactional/ICE/46espanol/ $\underline{46 \mathrm{ws} 5 \mathrm{~s} . \mathrm{htm}}$

Universidad de Costa Rica. Estatuto Orgánico. San José. Costa Rica. 1998. 
